\title{
Mesoporous Silica Loaded with Molybdenum Phosphide Nanoparticles for Hydrogen Evolution
}

\author{
Keliang Wang ${ }^{\mathbb{D}}$, Chan Jiang, Zhouyang Chen, and Shaojie Ma \\ Guangdong Polytechnic of Environmental Protection Engineering, Foshan 528216, China \\ Correspondence should be addressed to Keliang Wang; liangwk12@163.com
}

Received 4 July 2017; Accepted 19 October 2017; Published 21 January 2018

Academic Editor: Wei Zhou

Copyright (c) 2018 Keliang Wang et al. This is an open access article distributed under the Creative Commons Attribution License, which permits unrestricted use, distribution, and reproduction in any medium, provided the original work is properly cited.

A mesoporous silica loaded with molybdenum phosphide nanoparticles (MoP@MSN) was synthesized using Pluronic F-127 as a hard template. Using the method of XRD and HRTEM, the crystallinity, the phase structure, and the morphologies of the MoP@ MSN were investigated. The results showed that the MoP@MSN were composed of nanoflakes with approximately $100 \mathrm{~nm}$. Through the linear sweep voltammetry (LSV), the Tafel slopes of 100 and $341 \mathrm{mV}$ were yielded for MoP@MSN and pure MoP, respectively, meaning that the composite of MSN can significantly improve the conductivity of the products. Meanwhile, the mesoporous MoP@MSN presented excellent electrochemical activity and stability toward hydrogen evolution compared with those of bulk MoP nanoparticles, showing a promising prospect in hydrogen production.

\section{Introduction}

Hydrogen, a clean energy, has been considered as the new and sustainable energy source instead of the deficient fossil fuels in the future [1-3]. Electrochemical water splitting has been regarded as a potential way to produce $\mathrm{H}_{2}$, but this method might use electrocatalysts and consume plentiful power for the hydrogen evolution reaction (HER) $[4,5]$. This has induced intense researches to identify possible electrocatalysts that are suitable for HER, which at the moment is best catalyzed by noble metals, especially platinum $[6,7]$. However, the high cost of the noble metals has hindered their large-scale commercial application [8]. As a consequence, the research of low-cost alternatives made from affluent elements has become a main research focus for the practical application of $\mathrm{H}_{2}$.

The molybdenum-based compounds, a big family of non-noble-metal electrocatalysts, have received great success for generating hydrogen from water, including MoS [9-18], $\mathrm{Mo}_{2} \mathrm{C}$ [19-21], $\mathrm{NiMoN}_{\mathrm{x}}$ [22], and $\mathrm{Co}_{0.6} \mathrm{Mo}_{1.4} \mathrm{~N}_{2}$ [23]. Among them, molybdenum phosphide (MoP), an efficient HER catalyst in acids, has caused numerous attentions. Moreover, it has been confirmed that the turnover frequency (TOF) of MoP is much higher than that of $\mathrm{MoS}_{2}$, meaning better catalytic activity [24]. As we all know, an ideal catalyst should own a large specific surface area (SSA) for the exposure of more active sites and high electrical conductivity for favorable electron transfer [25]. As an abundant and lowcost semiconductor, silicon has been widely used in the field of solar energy conversion, including solar cell and photoelectrochemical water splitting devices $[26,27]$. Compared with the one-dimensional and two-dimensional structures, the three-dimensional (3D) porous morphology of silicon can provide a higher SSA and more catalytic sites, leading to superior catalytic activities [28].

In this article, a handy method for the synthesis of the composite of mesoporous silicon nanoparticle (MSN) and molybdenum phosphide (MoP) is introduced, and the remarkably enhanced hydrogen production capability of the resultant MoP@MSN has been demonstrated. In addition, we also explain the formation of MoP@MSN and the catalytic activities for hydrogen evolution.

\section{Materials and Methods}

2.1. Fabrication of $M o P$. Typically, $0.662 \mathrm{~g}\left(\mathrm{NH}_{4}\right)_{6} \mathrm{Mn}_{7} \mathrm{O}_{24}$ and $0.775 \mathrm{~g}\left(\mathrm{NH}_{4}\right)_{2} \mathrm{HPO}_{4}$ were dissolved in $15 \mathrm{~mL} \mathrm{H}_{2} \mathrm{O}$, with stirring at $90^{\circ} \mathrm{C}$ for $12 \mathrm{~h}$. After complete dissolution, with the 
evaporation at $120^{\circ} \mathrm{C}$ for $12 \mathrm{~h}$, a white solid can be obtained. Then, we calcine the white solid at $500^{\circ} \mathrm{C}$ for $5 \mathrm{~h}$ to get a green powder. In order to get the final target product, calcine the green powder at $850^{\circ} \mathrm{C}$ for $2 \mathrm{~h}$ in an atmosphere $\left(\mathrm{H}_{2} / \mathrm{Ar}=20 \%\right)$ to obtain a black powder S1.

2.2. Fabrication of MSN. Typically, $1.22 \mathrm{~g}$ Pluronic F-127 and $2.6 \mathrm{~g} \mathrm{CTAC}$ were added into $30 \mathrm{~mL} \mathrm{H}_{2} \mathrm{O}$ under stirring to get a clarified solution. Then, $4 \mathrm{~mL}$ TEOS was added into the solution with stirring for $24 \mathrm{~h}$, adding $30 \mathrm{~mL} \mathrm{NH} \cdot \mathrm{H}_{2} \mathrm{O}$ to get a white gel. The white gel was collected by centrifugation and washed with deionized water and ethanol and then dried at $60^{\circ} \mathrm{C}$ in an oven. Lastly, the product was calcined at $600^{\circ} \mathrm{C}$ for $3 \mathrm{~h}$ to obtain the final sample S2.

2.3. Fabrication of MoP@MSN. Typically, $0.662 \mathrm{~g}$ $\left(\mathrm{NH}_{4}\right)_{6} \mathrm{Mn}_{7} \mathrm{O}_{24}$ and $0.775 \mathrm{~g}\left(\mathrm{NH}_{4}\right)_{2} \mathrm{HPO}_{4}$ were dissolved in $15 \mathrm{~mL} \mathrm{H}_{2} \mathrm{O}$ by adding the as-synthesized MSN and then stirring at $90^{\circ} \mathrm{C}$ for $12 \mathrm{~h}$. After stirring, the clarified solution was oven-dried at $120^{\circ} \mathrm{C}$ for $12 \mathrm{~h}$, and a white solid could be obtained. Then, calcine the white solid at $500^{\circ} \mathrm{C}$ for $5 \mathrm{~h}$ and calcine the green powder at $850^{\circ} \mathrm{C}$ for $2 \mathrm{~h}$ in an atmosphere $\left(\mathrm{H}_{2} / \mathrm{Ar}=20 \%\right)$ to obtain a black powder. Lastly, the black powder was set in $2 \mathrm{M} \mathrm{NaOH}$ for $24 \mathrm{~h}$ to get the target product S3.

2.4. Sample Characterization. X-ray diffraction (XRD) patterns were collected on a Bruker D8 advanced X-ray diffractometer with $\mathrm{Ni}$-filtered $\mathrm{Cu} \mathrm{K} \alpha$ radiation $(\lambda=1.5406 \AA)$ at a voltage of $40 \mathrm{kV}$ and a current of $40 \mathrm{~mA}$. Transmission electron microscopic (TEM) images were taken on JEM-2010 and JEOL JEM-2100F microscopes.

2.5. Preparation of Working Electrodes. Catalyst ink was prepared by dispersing $1 \mathrm{mg}$ of catalyst in $900 \mu \mathrm{L}$ of ethanol solvent containing $100 \mu \mathrm{L}$ of $5 \mathrm{wt} . \%$ Nafion and sonicated for $60 \mathrm{~min}$. Then, $5 \mu \mathrm{L}$ of the catalyst ink was loaded onto a glassy carbon electrode (GCE) of $3 \mathrm{~mm}$ in diameter (loading ca. $0.72 \mathrm{mg} \cdot \mathrm{cm}^{-2}$ ).

2.6. Electrochemical Measurements. All the electrochemical measurements were conducted using a CHI1011 electrochemical workstation (CH Instruments, China) in a typical three-electrode setup with an electrolyte solution of $0.5 \mathrm{M}$ $\mathrm{H}_{2} \mathrm{SO}_{4}$, a Pt wire as the counterelectrode, and an Ag/AgClsaturated $\mathrm{KCl}$ as the reference electrode. Linear sweep voltammetry (LSV) was conducted in $0.5 \mathrm{M} \mathrm{H}_{2} \mathrm{SO}_{4}$ with a scan rate of $5 \mathrm{mV} \cdot \mathrm{s}^{-1}$. Onset overpotentials were determined based on the beginning of the linear regime in the Tafel plot. The time dependency of catalytic currents during electrolysis for the catalyst was tested in $0.5 \mathrm{M} \mathrm{H}_{2} \mathrm{SO}_{4}$ at $\eta=150 \mathrm{mV}$ after equilibrium. Electrochemical impedance spectroscopy (EIS, PARSTAT 2273, Princeton Applied Research, USA) measurements were carried out in the frequency range of $100 \mathrm{kHz}-$ $0.1 \mathrm{~Hz}$. Potentials were referenced to a reversible hydrogen electrode (RHE) by adding a value of $(0.197+0.059 \mathrm{pH}) \mathrm{V}$ [28].

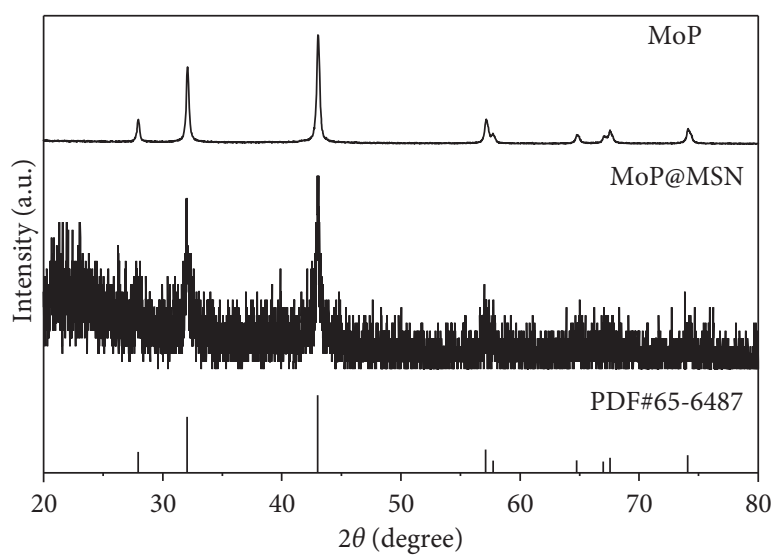

FIgURE 1: XRD patterns of MoP and MoP@MSN.

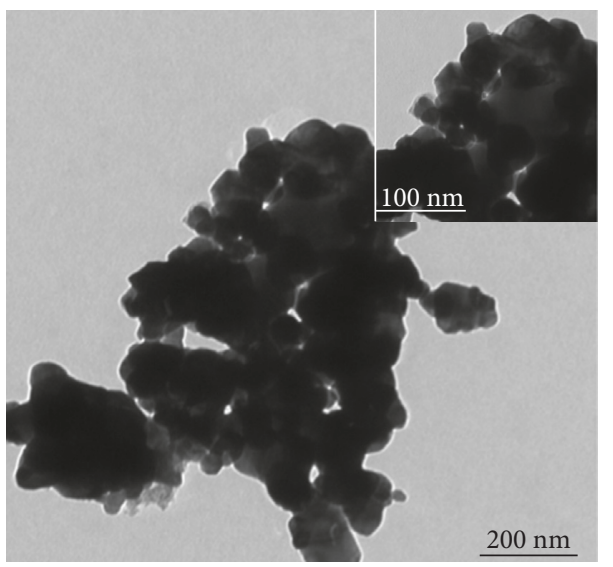

Figure 2: TEM of bulk MoP and HRTEM of bulk MoP (inset).

\section{Results and Discussion}

3.1. Characterization of Samples. Powder X-ray diffraction (XRD) was used to determine the crystallinity and phase structure of the as-synthesized products. As shown in Figure 1, the diffraction peaks of the as-synthesized sample S1 match well with those of MoP, meaning that $\mathrm{S} 1$ is a pure MoP (PDF\#65-6487) of good crystallinity. The diffraction peaks of S3 express low intensity, indicating a relatively low degree of crystallinity. Meanwhile, the diffraction peaks of S3 basically match with those of the pure MoP, and the discrepancy of peaks could be attributed to the amorphous phase of the MSN.

Transmission electron microscope (TEM) was used to investigate the morphologies of the samples. TEM images expressed the morphology and size of S1 and S3, as shown in Figures 2 and 3, respectively. Due to the high reaction temperature, the small particles gathered into clusters. Obviously, from Figure 2, we can easily find that the bulk $\mathrm{MoP}$ is stacked together, and the measure of the bulk MoP is about $600 \mathrm{~nm}$, consisting of the single nanosheets $(\sim 200 \mathrm{~nm})$. From the inset, the bulk MoP is composed of nanoflakes with approximately $100 \mathrm{~nm}$. Compared with the bulk MoP, Figure 3 showed that the phosphorization of MoP@MSN results in crystalline MoP on the sidewall of MSN. From Figure 3, we can 


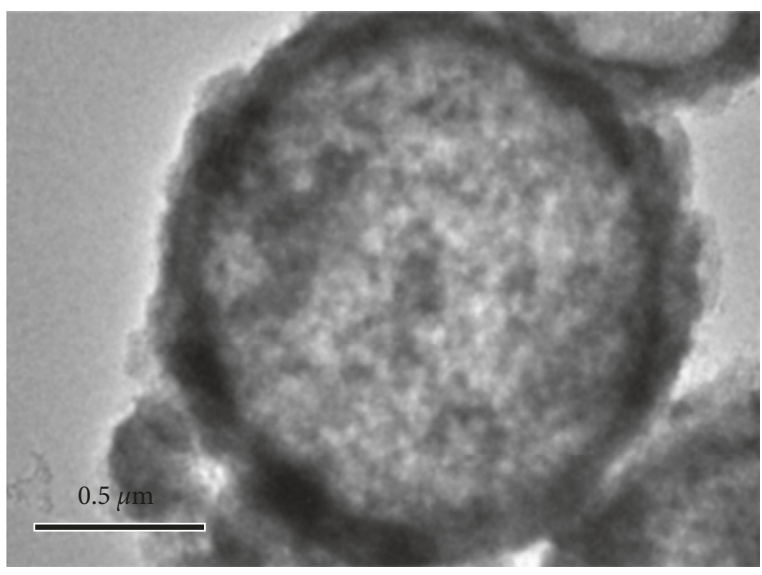

(a)

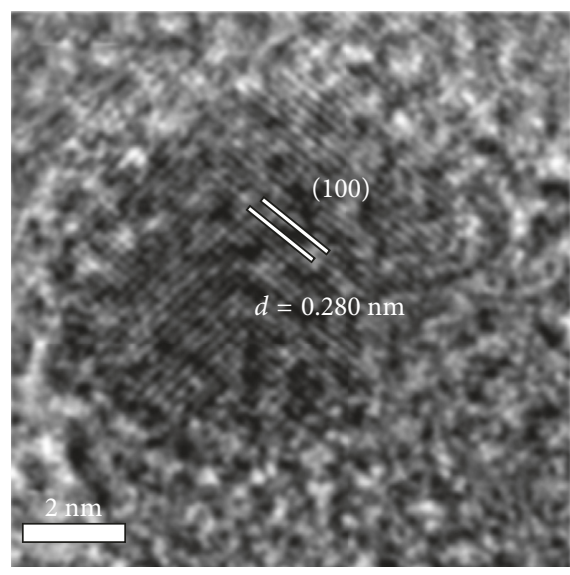

(b)

FIGURE 3: (a) TEM of MoP@MSN and (b) HRTEM of MoP@MSN.

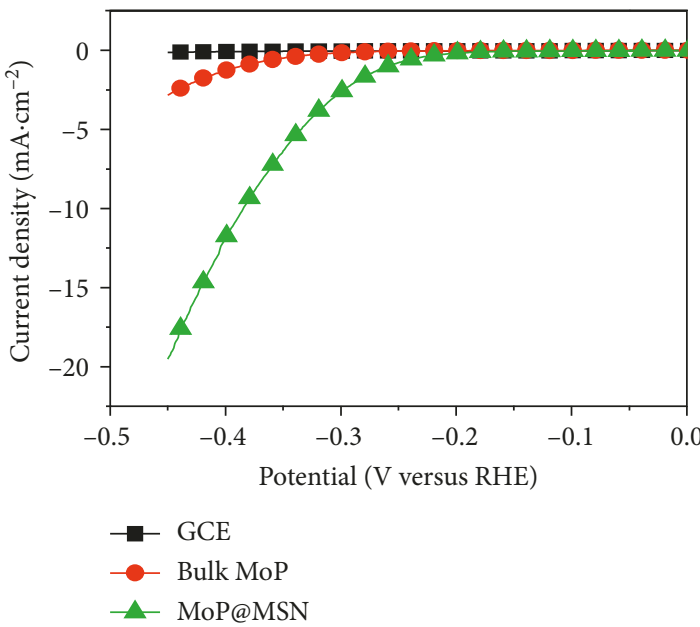

(a)

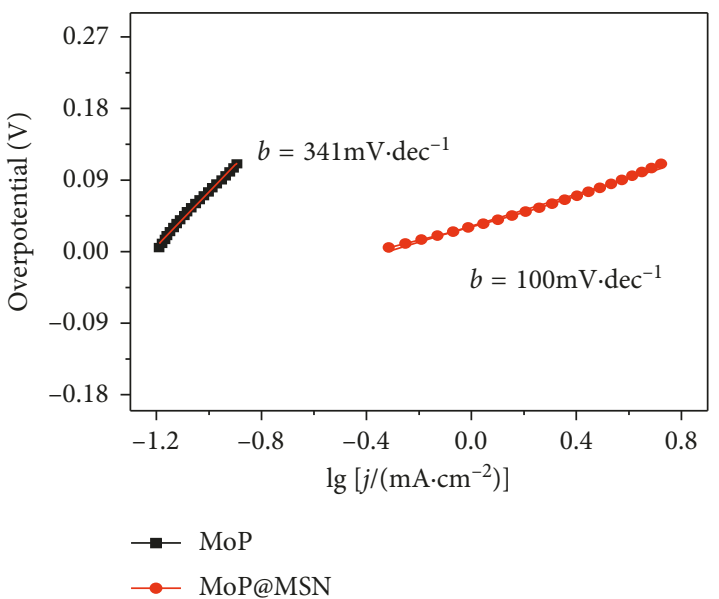

(b)

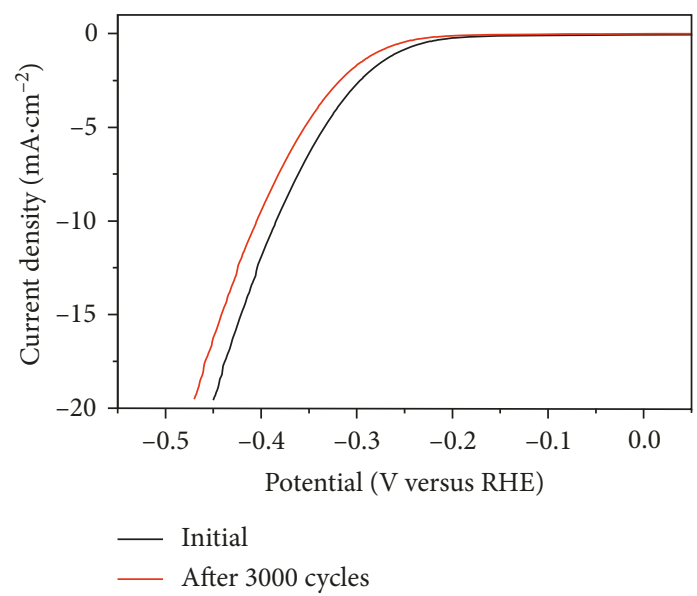

(c)

Figure 4: (a) Polarization curves of GCE, pure MoP, and MoP@MSN; (b) the Tafel plots of MoP and MoP@MSN after iR correction; (c) the stability test of the MoP@MSN catalyst.

also consider that the thin layer of $\mathrm{MoP}$ is constructed by thin open shells with a typical thickness of ca. $125 \mathrm{~nm}$. The highresolution TEM (HRTEM) image (Figure 3) shows clear lattice fringes with an interplane distance of $0.280 \mathrm{~nm}$, which correspond to the (100) plane of MoP, and the results were similar with the recent study [29]. 
3.2. Electrocatalytic Measurements. The electrocatalytic HER activity of MoP@MSN was confirmed through linear sweep voltammetry (LSV) in $\mathrm{H}_{2} \mathrm{SO}_{4}$ solution $(0.5 \mathrm{M})$ using a typical three-electrode setup. For comparison, a bare GCE and bulk MoP were used to experiment. As shown in Figure 4(a), the bare GCE and the bulk MoP expressed poor HER performance. The MoP@MSN displayed much higher cathodic current density for the HER, suggesting that the MoP@MSN is active for HER catalysis. The Tafel plots of the MoP and MoP@ MSN catalysts are displayed in Figure 4(b), which were fitted to the Tafel equation $(Z=b \log j+a$, where $j$ is the current density and $b$ is the Tafel slope), yielding the Tafel slopes of 100 and $341 \mathrm{mV}$ for MoP@MSN and pure MoP, respectively. The Tafel slope is regarded as an internal property of the catalyst, which is a significant standard for the HER performance. A smaller Tafel slope means a faster increase of the HER rate with increasing potential. Therefore, the MoP@MSN catalyst exhibits a better catalytic activity than the pure MoP catalyst, which can be ascribed to the lower Tafel slope of the MoP@MSN. In the process of electrocatalytic hydrogen evolution, the MSN offers specific surface area and catalytically active sites. In addition, the lower Tafel slope and the more positive onset potential for MoP@MSN might be possibly a result of the HER occurring on a porous matrix with higher SSA [30, 31].

Stability is a key factor in evaluating catalyst performance. The stability of the catalyst in an acidic environment was tested via 3000 cyclic voltammetric $(\mathrm{CV})$ cycles between 0 and $0.5 \mathrm{~V}$ versus the reversible hydrogen electrode (RHE) at $5 \mathrm{~m} \cdot \mathrm{Vs}^{-1}$. As shown in Figure 4(c), this electrode shows only a little change after 3000 cycles, compared with the first cycle of cathodic current density. The excellent catalytic performance of MoP@MSN might be attributed to the synergistic effects among the conductive carbon-modified and the porous structures. On one hand, MoP is a good conductor of electricity, which leads to fast electron transport across each microstructure and enhances electron penetration through the carbon layer. On the other hand, the porous structures of MSN offer a higher SSA and thus more active sites, which not only maximize the number of the exposed catalytic active sites but also facilitate the diffusion of the electrolyte and the release of generated gas bubbles [32].

\section{Conclusions}

In this work, we successfully synthesized a new kind of mesoporous silica loaded with molybdenum phosphide nanoparticles in a facile method, which showed a unique mesoporous structure and excellent stability. The MoP@ MSN is highly active for electrochemically generating hydrogen in acidic electrolytes. Such MoP@MSN offers an attractive low-cost metal catalyst material for the HER. We believe that our approach can be further extended to produce a series of carbon-coated and porous architectures containing transition metal phosphides for applications.

\section{Conflicts of Interest}

The authors declare that they have no conflicts of interest.

\section{Acknowledgments}

The authors would like to gratefully acknowledge the financial support by the Science and Technology Planning Project of Guangdong Province of China (no. 2014A020216052) and Science and Technology Innovation Fund of Foshan City of Guangdong Province of China (nos. 2015AG100242 and 2016AG100522).

\section{References}

[1] N. Armaroli and V. Balzani, "The hydrogen issue," Chemistry \& Sustainability, vol. 4, no. 1, pp. 21-36, 2011.

[2] D. G. Nocera, "The artificial leaf," Accounts of Chemical Research, vol. 45, no. 5, pp. 767-776, 2012.

[3] J. A. Turner, "Sustainable hydrogen production," Science, vol. 305, no. 5686, pp. 972-974, 1996.

[4] J. Wang, W. Cui, Q. Liu, Z. Xing, A. M. Asiri, and X. Sun, "Recent progress in cobalt-based heterogeneous catalysts for electrochemical water splitting," Advanced Materials, vol. 28, no. 2, pp. 215-230, 2016.

[5] M. G. Walter, E. L. Warren, J. R. McKone et al., "Solar water splitting cells," Chemical Reviews, vol. 110, no. 11, pp. 64466473, 2011.

[6] J. Greeley, T. F. Jaramillo, J. Bonde, I. Chorkendorff, and J. K. Norskov, "Computational high-throughput screening of electrocatalytic materials for hydrogen evolution," Nature Materials, vol. 5, no. 11, pp. 909-913, 2006.

[7] J. D. Holladay, J. Hu, D. L. King, and Y. Wang, "An overview of hydrogen production technologies," Catalysis Today, vol. 139, no. 4, pp. 244-260, 2009.

[8] J. Tian, Q. Liu, A. M. Asiri, and X. Sun, "Self-supported nanoporous cobalt phosphide nanowire arrays: an efficient $3 \mathrm{D}$ hydrogen-evolving cathode over the wide range of $\mathrm{pH}$ 0-14," Journal of American Chemical Society, vol. 136, no. 21, pp. 7587-7590, 2014.

[9] D. Merki and X. Hu, "Recent developments of molybdenum and tungsten sulfides as hydrogen evolution catalysts," Energy \& Environmental Science, vol. 4, no. 10, pp. 3878-3888, 2011.

[10] J. Kibsgaard, Z. Chen, B. N. Reinecke, and T. F. Jaramillo, "Engineering the surface structure of $\mathrm{MoS}_{2}$ to preferentially expose active edge sites for electrocatalysis," Nature Materials, vol. 11, no. 11, pp. 963-969, 2012.

[11] J. D. Benck, Z. Chen, L. Y. Kuritzky, A. J. Forman, and T. F. Jaramillo, "Amorphous molybdenum sulfide catalysts for electrochemical hydrogen production: insights into the origin of their catalytic activity," ACS Catalysis, vol. 2, no. 9, pp. 1916-1923, 2012.

[12] A. B. Laursen, P. C. Vesborg, and I. Chorkendorff, "A highporosity carbon molybdenum sulphide composite with enhanced electrochemical hydrogen evolution and stability," Chemical Communications, vol. 49, no. 43, pp. 4965-4967, 2013.

[13] J. Xie, H. Zhang, S. Li et al., "Defect-rich $\mathrm{MoS}_{2}$ ultrathin nanosheets with additional active edge sites for enhanced electrocatalytic hydrogen evolution," Advanced Materials, vol. 25, no. 40, pp. 5807-5813, 2013.

[14] D. Merki, H. Vrubel, L. Rovelli, S. Fierro, and X. Hu, "Fe, Co, and $\mathrm{Ni}$ ions promote the catalytic activity of amorphous molybdenum sulfide films for hydrogen evolution," Chemical Science, vol. 3, no. 8, pp. 2515-2525, 2012.

[15] T. F. Jaramillo, K. P. Jørgensen, J. Bonde, J. H. Nielsen, S. Horch, and I. Chorkendorff, "Identification of active edge 
sites for electrochemical $\mathrm{H}_{2}$ evolution from $\mathrm{MoS}_{2}$ nanocatalysts," Science, vol. 317, no. 5834, pp. 100-102, 2007.

[16] Z. Chen, D. Cummins, B. N. Reinecke, E. Clark, M. K. Sunkara, and T. F. Jaramillo, "Core-shell $\mathrm{MoO}_{3}-\mathrm{MoS}_{2}$ nanowires for hydrogen evolution: a functional design for electrocatalytic materials," Nano Letters, vol. 11, no. 10, pp. 4168-4175, 2011.

[17] D. Kong, J. J. Cha, H. Wang, H. R. Lee, and Y. Cui, "First-row transition metal dichalcogenide catalysts for hydrogen evolution reaction," Energy \& Environmental Science, vol. 6, no. 12, pp. 3553-3558, 2013.

[18] Y. H. Chang, C. T. Lin, T. Y. Chen et al., "Highly efficient electrocatalytic hydrogen production by $\mathrm{MoS}_{\mathrm{x}}$ grown on graphene-protected 3D Ni foams," Advanced Materials, vol. 25, no. 5, pp. 756-760, 2013.

[19] W. F. Chen, C. H. Wang, K. Sasaki et al., "Highly active and durable nanostructured molybdenum carbide electrocatalysts for hydrogen production," Energy \&Environmental Science, vol. 6, no. 3, pp. 943-951, 2013.

[20] H. Vrubel and X. Hu, "Molybdenum boride and carbide catalyze hydrogen evolution in both acidic and basic solutions," Angewandte Chemie International Edition, vol. 124, no. 51, pp. 12875-12878, 2012.

[21] L. Liao, S. Wang, J. Xiao et al., "A nanoporous molybdenum carbide nanowire as an electrocatalyst for hydrogen evolution reaction," Energy \& Environmental Science, vol. 7, no. 1, pp. 387-392, 2013.

[22] W. F. Chen, K. Sasaki, C. Ma et al., "Hydrogen-evolution catalysts based on non-noble metal nickel-molybdenum nitride nanosheets," Angewandte Chemie International Edition, vol. 51, no. 25, pp. 6131-6135, 2012.

[23] B. Cao, G. M. Veith, J. C. Neuefeind, R. R. Adzic, and P. G. Khalifah, "Mixed close-packed cobalt molybdenum nitrides as non-noble metal electrocatalysts for the hydrogen evolution reaction," Journal of American Chemical Society, vol. 135, no. 51, pp. 19186-19192, 2014.

[24] V. M. L. Whiffen and K. J. Smith, "Hydrodeoxygenation of 4-methylphenol over unsupported $\mathrm{MoP}, \mathrm{MoS}_{2}$, and $\mathrm{MoO}_{\mathrm{x}}$ catalysts," Energy \& Fuels, vol. 24, no. 9, pp. 4728-4737, 2015.

[25] S. Han, Y. Feng, F. Zhang et al., "Metal-phosphide-containing porous carbons derived from an ionic-polymer framework and applied as highly efficient electrochemical catalysts for water splitting," Advanced Function Materials, vol. 25, no. 25, pp. 3899-3906, 2015.

[26] R. N. Dominey, N. S. Lewis, J. A. Bruce, D. C. Bookbinder, and M.S. Wrighton, "Improvement of photoelectrochemical hydrogen generation by surface modification of p-type silicon semiconductor photocathodes," Journal of American Chemical Society, vol. 104, no. 2, pp. 467-482, 1982.

[27] S. Y. Reece, J. A. Hamel, K. Sung et al., "Wireless solar water splitting using silicon-based semiconductors and earthabundant catalysts," Science, vol. 334, no. 6056, pp. 645648, 2011.

[28] M. Liu and J. Li, "Cobalt phosphide hollow polyhedron as efficient bifunctional electrocatalysts for the evolution reaction of hydrogen and oxygen," ACS Applied Materials \& Interfaces, vol. 8, no. 3, pp. 2158-2165, 2016.

[29] Z. Xing, Q. Liu, A. M. Asiri, and X. Sun, "Closely interconnected network of molybdenum phosphide nanoparticles: a highly efficient electrocatalyst for generating hydrogen from water," Advanced Materials, vol. 26, no. 32, pp. 5702-5707, 2014.

[30] B. E. Conway and B. V. Tilak, "Interfacial processes involving electrocatalytic evolution and oxidation of $\mathrm{H}_{2}$, and the role of chemisorbed H," Electrochimca Acta, vol. 47, no. 22, pp. 3571-3594, 2002.

[31] Z. Lu, H. Zhang, W. Zhu et al., "In situ fabrication of porous $\mathrm{MoS}_{2}$ thin-films as high-performance catalysts for electrochemical hydrogen evolution," Chemical Communications, vol. 49, no. 68, pp. 7516-7518, 2013.

[32] X. Wang, Y. V. Kolen'ko, X. Q. Bao, K. Kovnir, and L. Liu, "One-step synthesis of self-supported nickel phosphide nanosheet array cathodes for efficient electrocatalytic hydrogen generation," Angewandte Chemie International Edition, vol. 54, no. 28, pp. 8188-8192, 2015. 


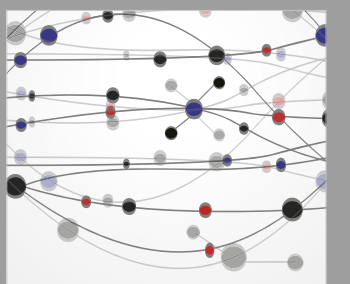

The Scientific World Journal
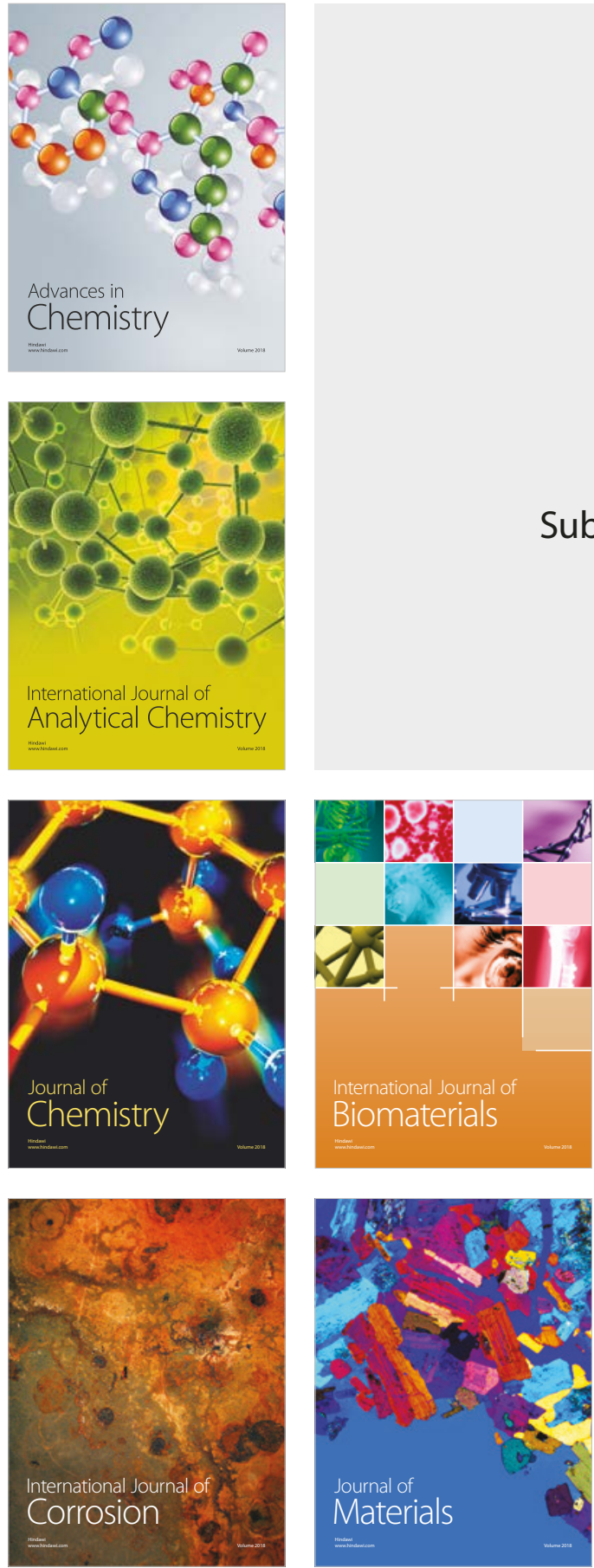

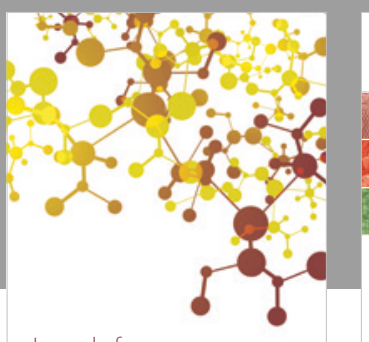

Journal of

Applied Chemistry
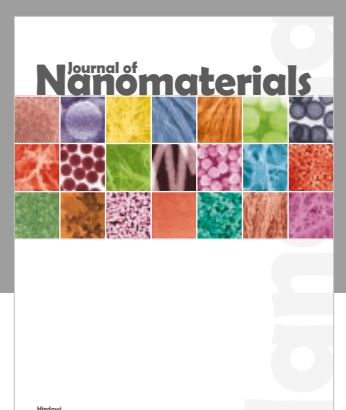

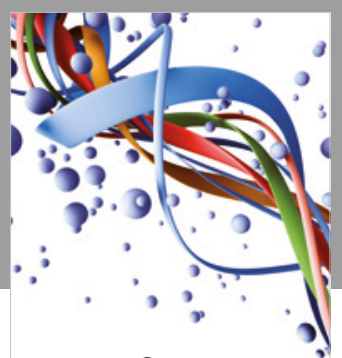

Scientifica

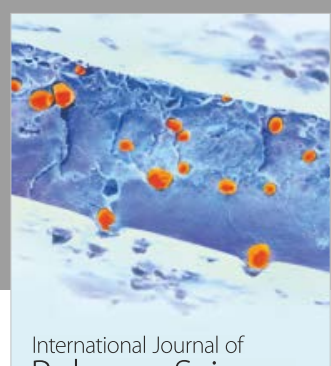

Polymer Science

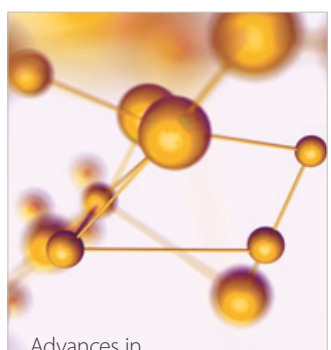

Physical Chemistry
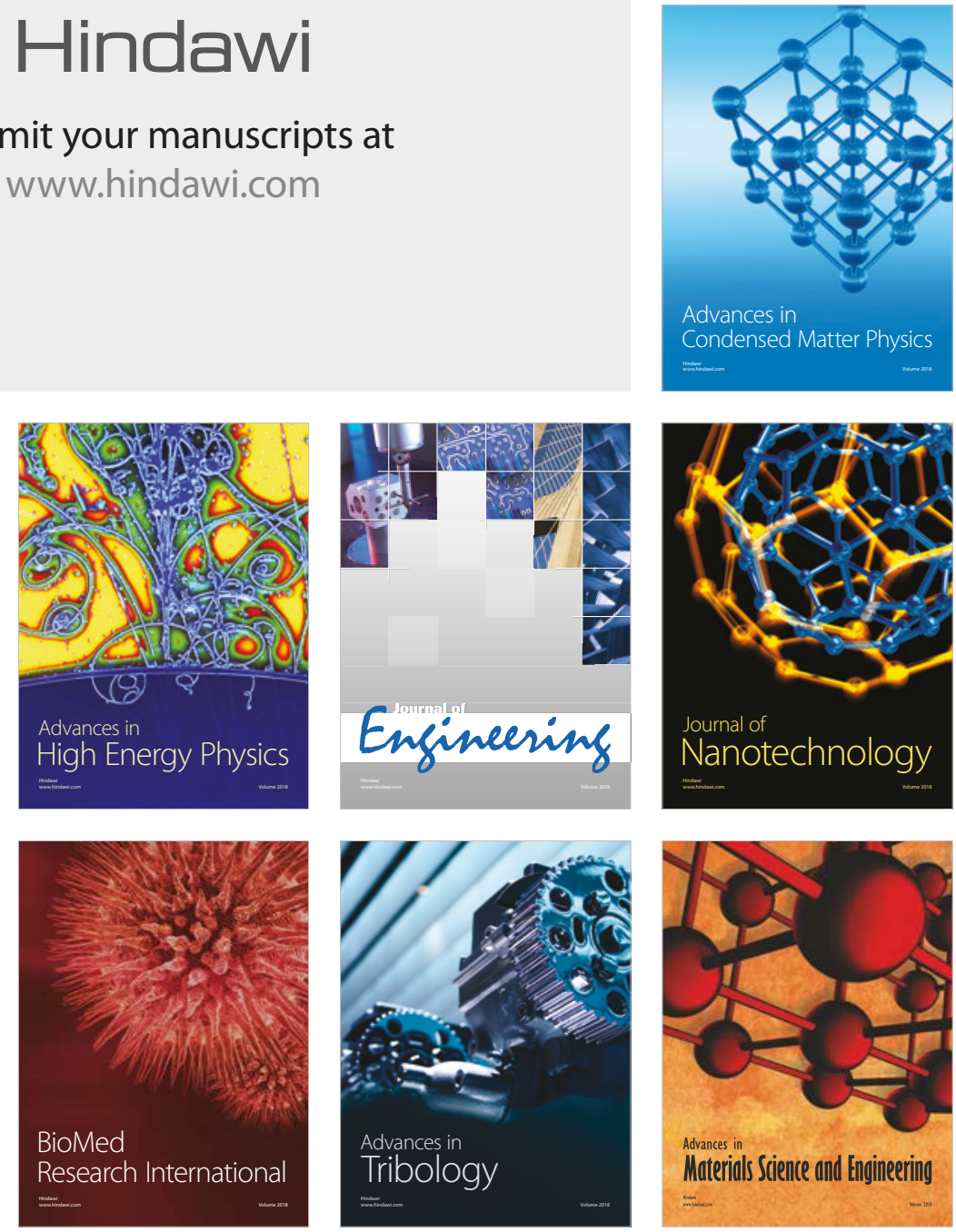